\title{
Perfil del profesor de inglés en Chile: quién es y qué enseña
}

\author{
Andrea Lizasoain \\ Universidad Austral de Chile. Chile. \\ andrea.lizasoain@uach.cl
}

Recibido: 3/12/2019

Aceptado: $13 / 7 / 2020$

Publicado: 29/1/2021

\section{Resumen}

El inglés ha tenido un papel relevante en Chile desde sus inicios como nación y durante cerca de doscientos años. En este contexto se intenta perfilar la imagen del profesor de inglés chileno para otorgarle el lugar que se merece y contribuir a hacer comprender el estado actual de dicha disciplina como lengua extranjera (ILE). Para ello, se realizó una encuesta en línea a un número estadísticamente significativo de profesores de inglés chilenos. Los resultados obtenidos se cotejaron con la base de datos del Ministerio de Educación (Mineduc) para validar la información por comparación externa. Se encontró que la mayoría de los docentes son mujeres jóvenes con niveles de proficiencia lingüística y formación pedagógica adecuados, con pocos años de experiencia y que enseñan la lengua inglesa por vocación para que los estudiantes ingresen en la fuerza laboral y amplíen sus visiones del mundo. Esto da cuenta de la influencia de las políticas lingüísticas en el currículo escolar y de formación docente, así como de la calidad de los profesores de inglés chilenos. Es decir, se logra el propósito de reivindicar su imagen por medio de datos empíricos.

Palabras clave: perfil del profesor; formación profesional docente; inglés como lengua extranjera; ILE; Chile

\section{Resum. Perfil del professor d'anglès a Xile: qui és i què ensenya}

L'anglès ha tingut un paper rellevant a Xile des del seu començament com a nació i gairebé al llarg de dos-cents anys. En aquest context s'intenta perfilar el professor d'anglès xilè per atorgar-li el lloc que es mereix i contribuir a fer comprendre l'estat actual d'aquesta disciplina com a llengua estrangera (ALE). Per aquest motiu es va realitzar una enquesta en línia a un nombre estadísticament significatiu de professors d'anglès xilens. Els resultats obtinguts es van confrontar amb la base de dades del Ministeri d'Educació per validar la informació per comparació externa. Es va trobar que la majoria dels docents són dones joves amb nivells de competència lingüística i formació pedagògica adequats, amb pocs anys d'experiència, que ensenyen la llengua anglesa per vocació perquè els estudiants entrin en el món laboral i ampliïn les seves visions del món. Això dona compte de la influència de les polítiques lingüístiques en el currículum escolar i de formació docent, com també de la qualitat dels professors d'anglès xilens. És a dir, s'aconsegueix el propòsit de reivindicar la imatge del professor d'anglès a Xile per mitjà de dades empíriques.

Paraules clau: perfil de professor; formació professional docent; anglès com a llengua estrangera; ALE; Xile 


\title{
Abstract. Profile of the English Teacher in Chile: Who are They and What do They Teach?
}

English has played an important role in Chile for almost 200 years. In this context, a profile of the Chilean English teacher is outlined to give them their rightful place and contribute to the understanding of the English as a Foreign Language discipline as it currently stands. An online questionnaire was sent to a statistically significant number of Chilean English teachers. Results were compared to a database provided by the Ministry of Education for comparative validation. It was found that most teachers are young women with adequate linguistic proficiency and training, with little experience, who teach English out of vocation for their students to enter the work force and to give them a broader view of the world. This reflects how Chilean linguistic policies influence the school and teacher training curricula and also highlights the teaching quality of Chilean English teachers. In other words, the purpose of vindicating the image of the Chilean English teacher is accomplished based on empirical data.

Keywords: teacher profile; professional teacher training; English as a Foreign Language; EFL; Chile

\author{
Sumario \\ 1. Introducción 4. Análisis y discusión \\ 2. Problematización 5. Conclusión \\ 3. Metodología Referencias bibliográficas
}

\section{Introducción}

La lengua inglesa ha tenido un papel relevante en el mundo desde el siglo Xvi, con los inicios del Imperio británico. En Chile, su influencia comenzó cuando este país se independizó de España y se instauró la república, en 1810. En consecuencia, resulta pertinente estudiar el rol del inglés en el desarrollo nacional. Este trabajo busca responder a la pregunta «icuál es el perfil del profesor de inglés chileno?» bajo la premisa de que el profesor es responsable de la educación que reciben los ciudadanos y, por ende, del desarrollo de una nación.

La contingencia nacional - e internacional - da cuenta de una crisis permanente de la educación pública, que ha llevado a los ciudadanos chilenos de todos los colores políticos a las calles a exigir una formación de calidad, desmarcada de la ideología neoliberal de mercado. Esta falta de calidad tiene relación con las malas condiciones para la gestión escolar, los bajos resultados de aprendizaje que arroja el Sistema de Medición de la Calidad de la Educación (SIMCE) y la magra formación integral que reciben los estudiantes en términos valóricos, artísticos y deportivos (Torche, Martínez, Madrid y Araya, 2015).

La asignatura de inglés del currículo escolar no está exenta de este problema, lo que se refleja en el bajo dominio de la lengua inglesa por parte de los ciudadanos chilenos, pese a los esfuerzos que se vienen realizando sistemáticamente desde el año 2003, cuando se creó el Programa Inglés Abre Puertas (PIAP). El objetivo del programa era lograr que, al finalizar la enseñanza escolar, los chi- 
lenos fueran capaces de, por lo menos, comunicarse en interacciones básicas como las que exigen situaciones de viaje. Más de quince años después, pareciera que los esfuerzos han sido en vano. Las pruebas estandarizadas que ha aplicado el Estado chileno en enseñanza media desde 2010 sistemáticamente arrojan resultados que ubican a los estudiantes por debajo del nivel básico del Marco Común Europeo de Referencia para las Lenguas (MCER). A su vez, el Índice de Dominio del Inglés de la consultora Education First (EF EPI) — que mide la proficiencia en inglés de más de 40 países anualmente- nos ha ubicado en los niveles «bajo» a "moderado» por años (EF EPI, 2019; 2018; 2017).

Las escasas publicaciones realizadas acerca de los profesores chilenos de inglés revelan aspectos negativos sobre ellos (Abrahams y Silva, 2017; Vivanco, 2016; Ortega, 2014; Valencia y Manzi, 2011; Abrahams y Farías, 2010; Véliz, 2007), por dicho motivo se les considera los responsables de esta deficiencia. Se ha acusado la falta de docentes para cubrir los establecimientos de todo Chile, donde estarían enseñando profesores que no son de la especialidad o que no están adecuadamente preparados (Vivanco, 2016; Ortega, 2014; Véliz, 2007). Una de las falencias señaladas acerca de su preparación es el bajo nivel de proficiencia que muestran al finalizar sus programas de estudios: «las estadísticas sugieren que solo un $28 \%$ de los profesores puede hablar inglés con un nivel de proficiencia avanzado y que solo un $8 \%$ puede utilizar la lengua meta adecuadamente al enseñarla» (Ortega, 2014, p. 11). También se ha dicho que, a causa de una formación pedagógica inapropiada (Abrahams y Silva, 2017; Abrahams y Farías, 2010), la interacción en el aula de inglés es de baja calidad (Valencia y Manzi, 2011). Esta brecha impediría el diálogo entre el proceso de adquisición de la lengua extranjera de los futuros docentes y su preparación pedagógica para luego enseñarla (Martin, 2016). Producto de lo anterior, pese a que dominan la lengua, los profesores desconocerían estrategias de manejo de grupo, enseñanza del idioma extranjero y evaluación de la competencia comunicativa (Véliz, 2007). Un diagnóstico general de los profesores de inglés en América Latina señala las mismas falencias (Cronquist y Fiszbein, 2017).

Por fortuna, también existen publicaciones que indican que nuestros profesores sí poseen una preparación adecuada; no obstante, una vez en el aula, se encuentran con dificultades para las cuales no han sido preparados (Barahona, 2015a; Lizasoain y Ortiz de Zárate, 2014; McKay, 2003). En esta línea, Lizasoain, Ortiz de Zárate, Véliz, Luci y Rojas-Mora (2016) encontraron que, al contrario de lo que se da por sabido, los profesores de la asignatura de inglés de contextos rurales de la Región Metropolitana, por ejemplo, sí son de la especialidad, dominan dicha lengua y utilizan estrategias de enseñanza-aprendizaje adecuadas. Sus dificultades están en relación con la incoherencia entre el currículo y la realidad del aula, la falta de recursos didácticos, la escasez de capacitación por parte del Mineduc, etc. Este tipo de contradicciones no les permiten realizar su labor docente con propiedad, independientemente de su preparación. McKay (2003), por su parte, además de referirse a los profesores de inglés chilenos como "bilingües» y de valorar su labor como docentes de lengua extranjera por encima de la de un hablante nativo, aplaude la disposi- 
ción y la proactividad de estos para adaptar el currículo a las necesidades reales de los aprendientes.

La literatura señala que el estudio de la identidad de los profesores es fundamental para comprender la naturaleza de sus prácticas pedagógicas, así como para transformar su formación docente y contribuir al desarrollo de la sociedad (Archanjo, Barahona y Finardi, 2019; Ha Le, 2008). «Definir este perfil puede ayudar a los profesores a comprender cómo deben ser, qué conocimientos y habilidades necesitan y cómo pueden mejorar» (Ribosa, 2020, p. 77). Los estudios sobre esta identidad se abordan mayormente desde las creencias y las autopercepciones (Martin y Rosas-Maldonado, 2019; Lehman y Weber, 2015; Blázquez y Tagle, 2010; Díaz y Solar, 2010), puesto que existen muy pocos trabajos que describan el perfil de un profesional. Este estudio surge de la convicción de que el perfil del profesor de inglés chileno no ha sido descrito con objetividad ni justicia. Para reinvindicarlo, se presenta una aproximación a su perfil, generado a partir de los datos obtenidos en una encuesta realizada a cerca de 400 profesores de aulas escolares de todo Chile.

\section{Problematización}

Las actuales políticas lingüísticas de lenguas extranjeras en Chile permiten contextualizar la figura del docente de inglés. Las políticas lingüísticas corresponden a ideas, opiniones, concepciones, etc. que se tienen acerca de las lenguas en relación con un determinado contexto político (Lizasoain, 2017; Calvet, 2006; Mar-Molinero, 2000). En los países latinoamericanos, estas se anidan en el currículo escolar (Mar-Molinero, 2000, 2006), donde se determina el estatus de las lenguas que conviven en un territorio, adjudicándoles etiquetas como «lengua nacional», "primera lengua», «lengua extranjera», «lengua internacional», etc.

Actualmente, el inglés tiene el estatus de «lengua extranjera» en el currículo escolar chileno. Una lengua extranjera se desarrolla con posterioridad a la lengua materna y, por lo tanto, en un contexto diferente. Si la primera lengua se aprende en el hogar mediante la interacción con la familia o los cuidadores con el fin de comunicarse en la cotidianeidad, la lengua extranjera se adquiere en contextos formales como la escuela, con fines específicos relativos al desarrollo escolar, académico y profesional.

El inglés como lengua extranjera (ILE) se enseña en Chile desde principios del siglo XIX con la creación del primer liceo, el Instituto Nacional. El área de lenguas y literatura del plan curricular incluía gramática castellana, traducción del francés, traducción del inglés y principios de dicción, poesía y crítica literaria. El inglés se enseñaba para tener acceso a la profundidad del pensamiento de esa lengua (Henríquez, 1812). La política lingüística original, entonces, se basaba en la idea de que el conocimiento de otras lenguas favorecía la formación integral de las personas, enriqueciendo su visión del mundo mediante el contacto con otras culturas. Los idiomas se consideraban capital cultural: un conjunto de saberes que permitían a los individuos desempeñarse en ámbi- 
tos exclusivos (Salado-Rodríguez y Ramírez-Martinell, 2018; Bourdieu, 1991). El inglés, en particular, también se enseñaba para formar embajadores, empresarios y turistas (Lizasoain, 2017). Al inaugurarse la Universidad de Chile, en 1843 , se explicitó que el inglés tenía que enseñarse para acercar el país a Europa y a los Estados Unidos, modelos de desarrollo (Bello, 1843). La enseñanza-aprendizaje del inglés da un vuelco y se pone al servicio de lo que MarMolinero (2006) denomina la «fuerza de arriba» (el poder político y económico); se convierte así en capital simbólico, sugiriendo que su adquisición otorga prestigio, sofisticación y modernidad (Matear, 2008). Cuando se creó el Instituto Pedagógico, en 1889, se formó por primera vez a profesores de inglés bajo el Programa para la Enseñanza del Inglés (Vera, 1942), con el fin de formar profesores capaces de «enseñar los idiomas como manifestaciones vivas del espíritu» (Lenz, citado en Vera, 1942, p. 212). Es decir, el conocimiento de una lengua extranjera debía integrarse en la identidad del futuro profesor, de manera que le permitiera expresarse en toda su magnitud social. En consecuencia, siempre ha existido una tensión en la disciplina de ILE en Chile, ya que la lengua debe enseñarse teniendo en cuenta la comunicación internacional, el acceso al conocimiento, la participación en redes de información y el comercio global (British Council, 2015; Matear, 2008; King, 2007; McKay, 2003), pero también para enriquecer el desarrollo del ser humano.

Esta imagen del profesor de inglés prevalece hoy y se encuentra explicitada en los Estándares orientadores para carreras de pedagogía en inglés (Ministerio de Educación, 2014). En este documento gubernamental se establece que, en cuanto a su formación disciplinaria, el docente debe ante todo desenvolverse en inglés con un nivel de proficiencia lingüística $\mathrm{C} 1$, que otorga a los hablantes un «dominio operativo eficaz» (British Council, 2019) basado en los fundamentos de la lengua inglesa y la competencia pedagógica. Lo mismo se espera de los docentes de inglés de la mayoría de los países de América Latina (Cronquist y Fiszbein, 2017). Asimismo, debe ser un agente de cambio social, capaz de formar ciudadanos con la capacidad de dialogar con otras culturas y de pensar el mundo actual de manera crítica. Dichos estándares, entonces, relevan la lengua inglesa como capital cultural y simbólico.

Un problema que plantea la descripción de un perfil profesional con base en competencias es el tiempo, ya que las necesidades de la sociedad cambian a un ritmo mucho mayor que los documentos que las describen, de manera que una vez que los perfiles se han implementado en los programas de formación, ya están desactualizados (Gallardo, 2019). Además, esta descripción está en el papel, de modo que es necesario hacer una descripción del profesor de inglés chileno en base a datos empíricos.

\section{Metodología}

Se diseñó una encuesta —-herramienta típica de los estudios descriptivos- que posibilitara realizar una generalización a partir de los datos obtenidos (Wagner, 2013; Castañeda Jiménez, 2011; Creswell, 2009; Nunan, 1992; Duverger, 
1972). Se creó en Google Forms y está compuesta por dos secciones destinadas a recabar información objetiva y subjetiva (Wagner, 2013). La primera sección presenta preguntas cerradas relacionadas con el sexo, la edad, la residencia, la formación, la proficiencia, las preferencias didácticas, etc. de los encuestados. La segunda sección contempla preguntas abiertas que buscan comprender qué significa el inglés como conocimiento concreto: «Cuando usted enseña inglés a sus estudiantes, ¿qué les está enseñando exactamente?», "¿Por qué decimos que el inglés es una lengua extranjera en Chile?» o «¿Cuál cree usted que es la mejor manera de enseñar inglés?».

La representatividad de la muestra se calculó en términos probabilísticos (Nunan, 1992), estableciéndose que debía estar conformada por 373 encuestas para un universo de 14.700 profesores de inglés. Este universo fue proporcionado por el Mineduc. Se consideró un margen de error de un 5\% y un nivel de confianza de un $95 \%$. El muestreo se realizó por conveniencia, es decir, se envió la encuesta a todos los individuos disponibles a los que se tuvo acceso en un periodo determinado (Wagner, 2013), mediante correo electrónico o a través de redes sociales. Se recopiló un total de 412 encuestas, de las cuales se desecharon 34 por encontrarse incompletas, por lo que finalmente se analizaron 378. Con el fin de darles validez mediante comparación externa (Castañeda Jiménez, 2011), los resultados se compararon con la base de datos facilitada por el Ministerio de Educación.

Las preguntas cerradas arrojaron datos cuantitativos que se sistematizaron en ocurrencias y porcentajes. Las preguntas abiertas se «cerraron» (Castañeda Jiménez, 2011) mediante la identificación de «temas», categorías recurrentes que surgen de los datos desde un enfoque inductivo (Creswell, 2009; Ryan y Bernard, 2003) y que se identifican a través de repeticiones, metáforas, analogías, diferencias y similitudes, entre otros aspectos. Esta última información también se examinó con el software de análisis de corpus AntConc (Anthony, 2020), a través del cual se identificaron los ítems léxicos más frecuentes en las respuestas abiertas entregadas por los profesores de inglés.

\section{Análisis y discusión}

\section{1. ¿Quién es el profesor de inglés en Chile?}

La encuesta arrojó que el $78 \%$ de los docentes corresponde a mujeres, mientras que un $28 \%$ son hombres (gráfico 1), lo que se condice con la base de datos del Mineduc, que registra que tres de cada cuatro profesores son mujeres (72\%). Estos datos también coinciden con la tendencia mundial, ya que más del 85\% de los profesores de esta lengua son mujeres (cifras de 2018) (The World Bank, 2019; The Commonwealth, 2011). Cabe destacar que en los primeros años de enseñanza del inglés en Chile la mayor parte de los profesores eran hombres hablantes nativos del inglés (Vera, 1942). La «feminización» de la educación, en general, se asocia con su mayor cobertura en los países desarrollados, para lo cual las mujeres han jugado un papel fundamental (The Commonwealth, 2011). 
Gráfico 1. Distribución por sexo de la muestra

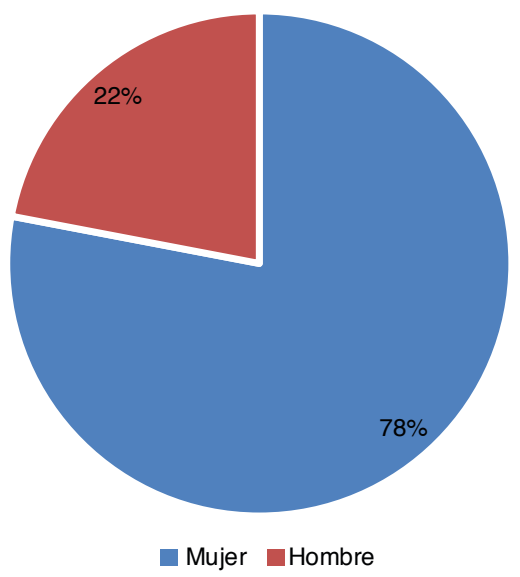

Fuente: Lizasoain (2018).

Con respecto al rango etario de los profesores, la mayor parte son menores de 30 años (un 41\%), seguido por aquellos que se encuentran entre los $30 \mathrm{y}$ los 39 años (un 31\%). Un porcentaje menor corresponde a profesores mayores de 50 años (un 15\%), lo cual es coherente con la información entregada por el Mineduc (gráfico 2). Su juventud se explica porque el Estado chileno ha lanzado iniciativas para aumentar el contingente de profesores de todas las áreas y mejorar la calidad de la educación (Ministerio de Educación, 2018). Desde 2011, la beca Vocación de Profesor permite estudiar carreras de peda-

Gráfico 2. Distribución etaria de la muestra

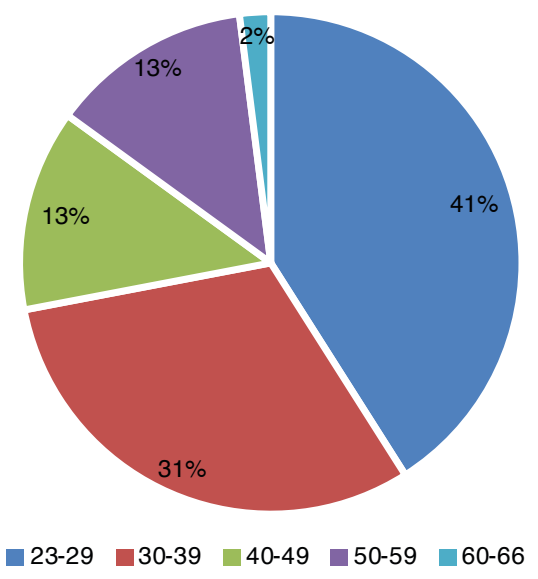

Fuente: Lizasoain (2018). 
gogía sin costos asociados, por lo que muchos jóvenes han decidido dedicarse a la enseñanza. En consecuencia, los docentes que actualmente se encuentran en las aulas han egresado recientemente de la universidad.

En concordancia con la edad de los profesores, la mayoría de estos (un 68\%) ha trabajado menos de 10 años, por lo que cuentan con poca experiencia en el aula. Cerca de un 30\% ha trabajado entre 10 y 40 años (gráfico 3). Estos resultados se condicen con la base de datos nacional, la cual registra que de los 14.700 docentes del país, 11.221 (un 76\%) tienen menos de 10 años de experiencia.

Gráfico 3. Distribución de la experiencia docente

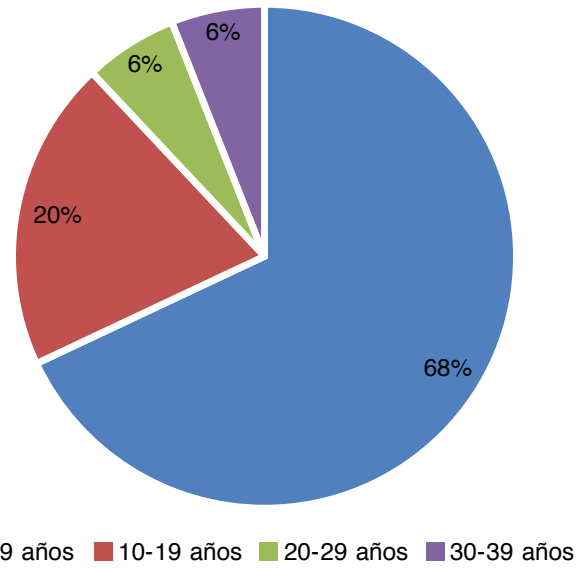

Fuente: Lizasoain (2018).

Es necesario señalar que no existe evidencia empírica que asegure que a mayor experiencia en docencia, mejor calidad de la enseñanza (Santelices, Galleguillos, González y Taut, 2015; Jacob y Legfren, 2002). De hecho, en Chile, la valoración del Sistema de Evaluación Docente aplicada por MideUC encontró que el 63\% de los profesores con más de 10 años de experiencia eran calificados como competentes y destacados, mientras que un promedio del $59 \%$ lo obtenía con menos de 10 años, diferencia que no resulta significativa (Valencia y Manzi, 2011).

En relación con la formación profesional, contrariamente a lo señalado por Vivanco (2016), el 78\% de los profesores cuenta con título profesional de inglés (gráfico 4), lo que se condice con el porcentaje que registra el Mineduc (un 68\%). El segundo grupo más robusto (un 16\%) lo conforman profesores con mención en inglés, los cuales han estudiado otras pedagogías y se han capacitado para enseñar esta lengua. El tercer grupo (un 6\%) está formado por profesionales habilitados por el Mineduc como traductores, y han sido autorizados para ejercer la pedagogía por su conocimiento de la lengua. Entonces, Chile cuenta con el contingente especializado necesario para enseñar inglés. 
Gráfico 4. Distribución del título profesional de la muestra

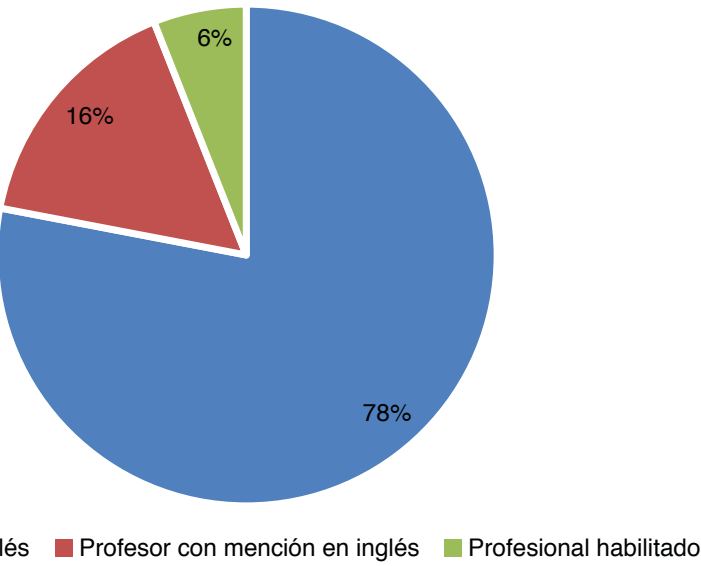

Fuente: Lizasoain (2018).

Uno de los requisitos que deben cumplir los profesores de inglés para ser valorados e integrados a sus comunidades de práctica es demostrar ser un hablante avanzado de la lengua inglesa (Barahona e Ibaceta, 2019; Lizasoain, 2019). Un nivel B2 de proficiencia indica que el hablante entiende la información más importante de textos complejos, ya sea que aborden temas concretos o abstractos. Los hablantes no nativos con este nivel pueden comunicarse tanto con hablantes nativos como no nativos, con grados de fluidez y naturalidad suficientes para la comunicación efectiva, al menos en el contexto de aula de enseñanza secundaria. Además, producen textos coherentes acerca de variados contenidos y pueden defender su punto de vista (Consejo de Europa, 2002). Este es el nivel esperado para la finalización de la educación escolar en Chile. El nivel C1 permite comprender y producir una amplia gama de textos extensos y complejos, con una exigencia pragmática más alta que en el caso del nivel B2. Un hablante no nativo con nivel C1 puede expresarse con naturalidad en cualquier contexto, no solo en los de su especialidad. Es el nivel máximo que suelen alcanzar los hablantes de lengua extranjera (Consejo de Europa, 2002).

El Mineduc ofrece la posibilidad de certificar el nivel de inglés a través de una prueba de proficiencia voluntaria. Solo cerca de un cuarto de los 14.700 profesores se ha certificado (un 28\%), por lo que la encuesta aplicada en esta investigación incluyó un ítem para conocer el nivel de inglés percibido de los profesores. Gran parte de ellos (un 45,5\%) se clasificó en el nivel B2, lo cual contrasta con la base de datos del Mineduc, que registra un 27\% de docentes con este nivel, un alto porcentaje de los cuales indicó que contaba con el nivel C1 (un 37\%), exigido a partir de 2014 por el Ministerio (gráfico 5).

En contraste, la base de datos gubernamental indica que solo un 19\% de los profesores se clasifica en el grado más alto exigido. Según Barahona e Ibaceta 
Gráfico 5. Distribución del nivel de competencia comunicativa

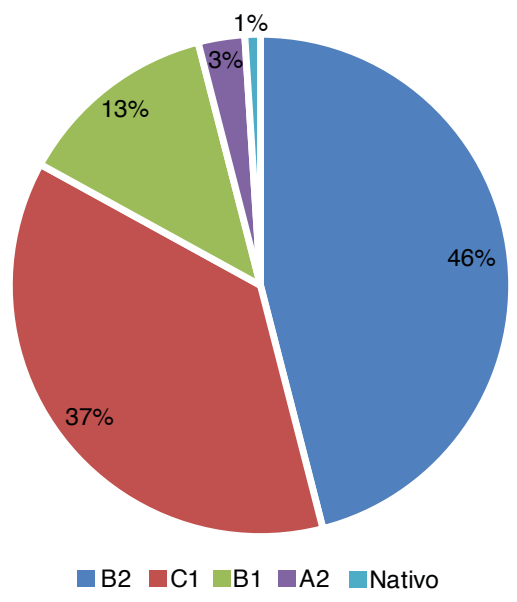

Fuente: Lizasoain (2018).

(2019), esta contradicción entre los niveles de proficiencia percibidos y los constatados mediante mediciones objetivas se puede comprender como «una aspiración [de los profesores de inglés] a cumplir con los estándares [del Ministerio de Educación] y, en consecuencia, satisfacer expectativas sociales» (p. 4).

Una de las formas de clasificar los establecimientos escolares chilenos es según los niveles socioeconómicos generales (bajo, medio y alto) que se establecen en el marco de las pruebas SIMCE (Sistema de Medición de Conocimientos Escolares). El nivel bajo agrupa a estudiantes cuyas familias tienen ingresos promedio entre 180 y 270 dólares; el nivel medio, entre 270 y 850 dólares, y el nivel alto, entre 850 y 2.200 dólares (Agencia de Calidad en la Educación, 2012) ${ }^{1}$. La encuesta arrojó que un alto porcentaje de los profesores se desempeña en establecimientos de nivel bajo (un 49\%), seguido del nivel medio (un 40\%) (gráfico 6). Esta realidad da cuenta de que los bajos resultados en las pruebas estandarizadas no tendrían que ver con la preparación de los docentes, sino con el tipo de establecimiento y el nivel socioeconómico de las familias. Es decir, mientras más desaventajados son los estudiantes, más difícil parece la tarea de enseñar y aprender. Archenjo, Barahona y Finardi (2019) proponen que los estudiantes más desaventajados tienen profesores con una proficiencia en inglés más baja que aquellos estudiantes más aventajados. Esto ocurre porque la proficiencia se va deteriorando si los hablantes de lenguas extranjeras dejan de usar sus recursos más avanzados, de manera que si un profesor trabaja en una escuela de pocos recursos, termina con un nivel de proficiencia más bajo, al no poder poner en práctica los recursos con los que contaba en un principio.

1. No se encontraron fuentes más actualizadas por parte de la Agencia de Calidad de la Educación chilena. 
Gráfico 6. Distribución del nivel socioeconómico de los establecimientos

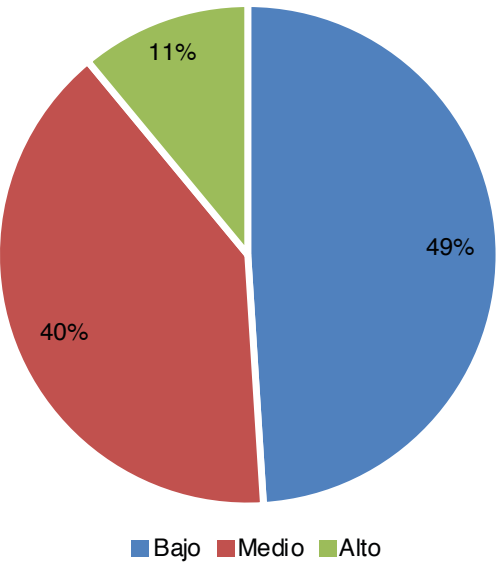

Fuente: Lizasoain (2018).

La encuesta incluyó un ítem para seleccionar las razones que mejor describieran por qué un profesor había escogido esta carrera (gráfico 7). Existen dos

Gráfico 7. Distribución de las razones para enseñar inglés

No lo tengo muy claro | 4

En mi escuela no hay profesores especialistas y me pidieron que lo enseñara yo

Creo que si los estudiantes no saben inglés, serán considerados analfabetos

Tengo facilidades para los idiomas y aproveché ese don para ganarme la vida

Me interesa que los estudiantes puedan entender las películas, series, videojuegos y otros que están en inglés

Creo que es importante que los estudiantes puedan comunicarse con los extranjeros que vienen a Chile

Considero importante que los estudiantes sepan inglés para poder viajar y conocer el mundo

Me gustan los idiomas

Me gusta enseñar idiomas

Busco que mis estudiantes amplíen su visión del mundo acercándose a otra cultura

Quiero que los niños de hoy tengan mejores oportunidades laborales en el futuro
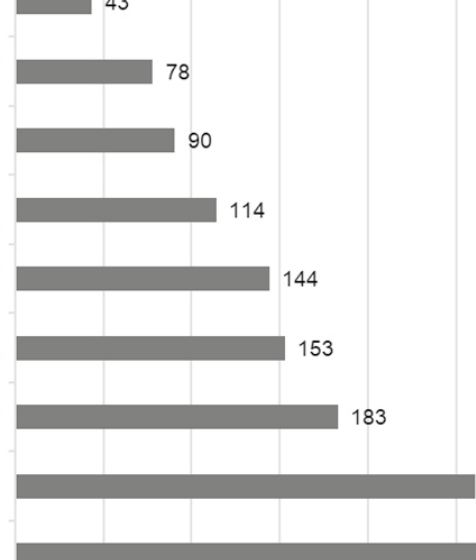

Fuente: Lizasoain (2018). 
grandes motivos: quieren que sus estudiantes puedan acceder a mejores trabajos (seleccionada 263 veces) y que se acerquen a otras culturas (seleccionada 261 veces). De esta manera, el inglés aparece como capital simbólico y cultural, lo que coincide con la visión del Mineduc de que dicho idioma se incluye en el currículo escolar para que los estudiantes puedan "comunicarse en situaciones similares a las de la vida real y acceder a diversos conocimientos que contribuyan a su vida académica o laboral futura» (Ministerio de Educación, 2015, p. 216). Estas respuestas reflejan las políticas lingüísticas chilenas, volcadas en el desarrollo económico y académico. Se ponen en el dominio del inglés las esperanzas de convertir a Chile en un país verdaderamente desarrollado. La responsabilidad otorgada al profesor de esta lengua, entonces, es grande.

Es importante destacar que los profesores también imparten dicha materia porque les gusta (seleccionada 183 veces) y porque es importante saber inglés para conocer el mundo (144). Es decir, actúan por verdadera vocación: les gustan los idiomas y también les gusta enseñarlos. Esto se puede correlacionar con las altas tasas de motivación con la que enseñan los profesores de inglés hoy (Archenjo, Barahona y Finardi, 2019; Barahona e Ibaceta, 2019), a pesar del desprecio social generalizado de que son víctimas (Ribosa, 2020; Barahona e Ibaceta, 2019; Bellei y Valenzuela, 2013).

\section{2. ¿Qué enseña el profesor de inglés en Chile?}

Se buscó saber cómo se representa el «inglés como lengua extranjera» en el discurso: qué es el inglés como conocimiento concreto y como constructo político. Se identificaron los ítems léxicos más frecuentes en las respuestas abiertas con el software AntConc (Anthony, 2020). Paralelamente, se realizó un análisis de contenido para identificar los temas recurrentes.

Los recursos lingüísticos más frecuentes en el corpus de respuestas abiertas fueron comunicación, cultura, habilidades, desarrollo, valores, gramática y vocabulario. Los temas identificados tuvieron relación con la idea de que el «inglés como lengua extranjera» se define como un idioma que se enseña con fines comunicativos y culturales. Esto se lleva a cabo mediante el desarrollo de habilidades y la enseñanza-aprendizaje de gramática y vocabulario, lo que se condice con la relevancia que otorgan las bases curriculares a estrategias de enseñanza-aprendizaje que promuevan la comunicación en lugar del conocimiento acerca de la lengua. En este sentido, enseñar inglés significa desarrollar habilidades de comunicación, en lugar de aprender listas de vocabulario y reglas gramaticales, como fue en el pasado. Las siguientes respuestas textuales de los profesores respaldan esta interpretación:

1. «Más que solo una lengua, enseño una cultura. Inglés no es solo pronunciar bien una palabra o saber la gramática de una oración, es ser capaz de entender diferentes culturas, costumbres, tradiciones e historia, para así también apreciar nuestra propia lengua y cultura.» 
2. «Les enseño a comunicarse. Les enseño a transmitir su mensaje más que a preocuparse por la estructura. También les enseño a amar el idioma y a darse cuenta de que cualquiera puede aprender inglés con paciencia y perseverancia.»

3. «En una herramienta comunicativa que les dará mejores y mayores oportunidades laborales, específicamente, a aquellos que decidan desempeñarse inmediatamente en el ámbito laboral al terminar la enseñana [sic] media.»

En suma, los resultados apuntan a que el perfil del profesor de inglés chileno característico es una mujer joven con niveles adecuados de proficiencia y de formación para impartir dicha materia, con pocos años de experiencia y cuya vocación es enseñar con fines comunicativos y culturales para mejorar la calidad de vida de sus estudiantes.

\section{Conclusión}

En este trabajo se definió un perfil del profesor de inglés en Chile con el fin de reivindicar su rol, necesario no solo en nuestra disciplina, sino también en la docencia en general. Pese al mal diagnóstico acerca de su calidad y de su efectividad, los profesores de inglés chilenos son competentes, lo que Ribosa (2020) define como profesionales con un alto dominio de su materia y su acción moral. Si bien este perfil no asegura la calidad de la enseñanza-aprendizaje de la lengua extranjera, un buen manejo del inglés - más formación inicial docente - es garantía de calidad pedagógica (Archenjo, Barahona y Finardi, 2019; Barahona e Ibaceta, 2019).

En consonancia con la historia del inglés en Chile y con el currículo nacional, se encontró que el profesor de esta especialidad concibe la lengua extranjera en dos términos. Por una parte, es una herramienta de comunicación que permite acceder a más y mejores oportunidades académicas y laborales. Y, por otra, es un capital cultural y simbólico que abre las puertas a otros mundos que no podrían conocerse sin el manejo de la lengua inglesa. Este tipo de subjetividad ejerce un impacto en el aula, porque predispone a los participantes a tener ciertas actitudes hacia el conocimiento, lo que «constituye un terreno fértil para estudiar y potenciar la implementación de distintos procesos de cambio y mejora en las escuelas» (Torche et al., 2015, p. 109). Por eso, es posible y deseable aprovechar la preparación y la vocación de los profesores de inglés por enseñar la lengua, para así mejorar los niveles de proficiencia de los chilenos, lo que puede lograrse generando cambios que faciliten su trabajo en el aula. Esto implica alejarse del modelo neoliberal de mercado que impera en la educación chilena y acercarse a una práctica que se ajuste a las necesidades actuales de las comunidades escolares y ciudadanas.

Por último, cabe destacar que no existe un único modelo ideal de profesor de inglés, porque este depende del contexto. Sin embargo, es importante describir los distintos perfiles para poder compararlos y contrastarlos con otros, 
así como rescatar enseñanzas para mejorarlos en pro de una mejor educación $y$, en concordancia, de una mejor calidad de vida.

\section{Referencias bibliográficas}

Abrahams, M. y Farías, M. (2010). Struggling for Change in Chilean EFL Teacher Education. Colombian Applied Linguistics Journal, 12(2), 110-118. <https://doi.org/10.14483/22487085.87>.

Abrahams, M.J. y Silva, P. (2017). What happens with English in Chile?: Challenges in Teacher Preparation. En L. Kamhi-Stein, G. Díaz Maggioli y L. de OliveiRA (eds.). English language teaching in South America, policy, preparation and practice (pp. 109-122). Bristol: Multilingual Matters.

Agencia de Calidad de la Educación (2012). Metodología de construcción de grupos socioeconómicos: Pruebas Simce 2012. Recuperado de <http://archivos.agenciaedu cacion.cl/Metodologia-de-Construccion-de-Grupos-Socioeconomicos-SIMCE2012.pdf>.

Anthony, L. (2020). Antconc para Windows 3.4.4. Recuperado el 16 de junio de 2020, de <http://www.laurenceanthony.net/software.html>.

Archanjo, R.; Barahona, M. y Finardi, K. (2019). Identity of foreign language pre-service teachers to speakers of other languages: Insights from Brazil and Chile. Colomb. Appl. Linguistic. J., 21(1), 62-75.

Barahona, M. (2015a). Contradictions in the activity of Learning to Teach English in Chile. En B. Selau y R. Fonseca. Cultural Historical Approach: Educational Research in Different Contexts (pp. 73-97). Porto Alegre: EDIPUCRS.

- (2015b). English Language Teacher Education in Chile: A Cultural Historical Actitvity Theory Perspective. Nueva York: Routledge.

Barahona, M. e Ibaceta-Quijanes, X. (2019). Neither Fish nor Fowl: The Contested Identity of Teachers of English in an EFL Context. RELC Journal, 1-17. <https://doi.org/10.1177/0033688219847315>

Bellei, C. y Valenzuela, J. (2013). El estatus de la profesión docente en Chile. Percepción de los profesores acerca del estatus profesional de la docencia. En B. Avalos (ed.). ¿Héroes o villanos? La profesión docente en Chile (pp. 1-32). Santiago de Chile: Editorial Universitaria.

Bello, A. (1843). Discurso inaugural. Universidad de Chile (17 de septiembre). Recuperado de <http://www.uchile.cl/portal/presentacion/historia/4682/discursoinaugural $>$.

Blázquez, F. y TAgLe, T. (2010). Formación docente: Un estudio de las creencias de alumnos y profesores sobre el proceso de enseñanza y aprendizaje del inglés. Revista Iberoamericana de Educación, 54(4), 1-12.

Bourdieu, P. (1991). Language and Symbolic Power. Cambridge: Polity/Blackwell.

British Council (2015). English in Chile: An Examination of Policy, Perceptions and Influencing Factors. Recuperado de <http://obiret-iesalc.udg.mx/sites/default/files/ publicaciones/44._english_in_chile.pdf>.

- (2019). Nivel de inglés C1. Recuperado el 19 de noviembre de 2019, de <https:// www.britishcouncil.es/ingles/niveles/c1>.

Calvet, L.J. (2006). Language Wars: Language Policies and Globalization. Nanovic Institute for European Studies. Recuperado de <https://nanovic.nd.edu/ assets/8706/calvetpaper.pdf>.

Castañeda Jiménez, J. (2011). Metodología de la investigación. México: McGraw-Hill. 
Consejo de Europa (2002). Marco Común Europeo de Referencia para las Lenguas: Aprendizaje, enseñanza y evaluación. Madrid: Ministerio de Educación, Cultura y Deporte. Subdirección General de Cooperación Internacional.

Creswell, J. (2009). Research Design: Qualitative, Quantitative, and Mixed Methods Approaches (3. ${ }^{\mathrm{a}}$ ed.). California: Sage.

Cronquist, K. y Fiszbein, A. (2017). El aprendizaje del inglés en América Latina. El Diálogo: Liderazgo para las Américas.

Díaz, C. y Solar, M. (2011). La revelación de las creencias lingüístico-pedagógicas a partir del discurso del profesor de inglés universitario. RLA: Revista de Lingüistica Teórica y Aplicada, 49(2), 57-86.

Duverger, M. (1972). Métodos de las ciencias sociales. Barcelona: Ariel.

EF EPI (2017). EF English Proficiency Index. Recuperado el 28 de noviembre de 2019, de <https:/www.ef.com/_/ //media/centralefcom/epi/downloads/full-reports/ v7/ef-epi-2017-spanish-latam.pdf>.

- (2018). EF Índice del dominio del inglés de EF. Recuperado el 28 de noviembre de 2019, de <https://www.ef.com/ /media/centralefcom/epi/downloads/fact-sheets/ v9/ef-epi-country-fact-sheet-v9-cl-es.pdf>.

- (2019). Hoja de datos: Chile. Recuperado el 27 de noviembre de 2019, de <https:// www.ef.com/ -/media/centralefcom/epi/downloads/fact-sheets/v9/ef-epi-countryfact-sheet-v9-cl-es.pdf>.

Gallardo, B. (2019). Perfil del docente en Bibliotecología en tres universidades de Buenos Aires. Información, Cultura y Sociedad, 41, 35-56. <https://doi.org/10.34096/ics.i41.6074>

Ha Le, P. (2008). Teacher Identity and the Teaching of English. En H.L. Phan. Teaching English as an international language: Identity, Resistance and Negotiation (pp. 178-195). Bristol: Equinox.

Henríquez, C. (1812). Bases para la creación del Instituto Nacional de Chile - Parte 2. Aurora de Chile (25 de junio). Recuperado de <http://www.auroradechile.cl/ newtenberg/681/article-2824.html>.

Jacob, B. y Legfren, L. (2002). The Impact of Teacher Training on Student Achievement: Quasi-experimental Evidence From School Reform Efforts in Chicago. Cambridge: National Bureau of Economic Research. Recuperado de <http://www.nber. org/papers/w8916.pdf>.

KING, P. (2007). Estudio multidimensional de la oralidad a partir de los textos escolares para la enseñanza de inglés como lengua extranjera. Revista Signos, 40(63), 101-126.

Lehman, T. y Weber, T. (2015). English-teachers' Teaching Perspectives and Their use of Methods to Foster Students' Communicative Competence: A Comparison Between Chile and Germany. The Journal of Language Teaching and Learning, 5(2), 22-36.

Lizasoain, A. (2017). El lugar del inglés en las políticas y planificación lingüísticas chilenas: ¿de dónde venimos y hacia adónde vamos? Lenguas modernas, 19, 121133.

- (2018). Construcción del conocimiento en el aula de Inglés como Lengua Extranjera en un contexto de enseñanza media en Chile: análisis del discurso pedagógico desde un enfoque sistémico funcional. Tesis para optar al grado de Doctor en Lingüística por la Pontificia Universidad Católica de Chile. Repositorio UC.

- (2019). Reconstrucción discursiva de la identidad de una profesora universitaria e incorporación a su comunidad de práctica. Logos, 29(2), 238-253. 
Lizasoain, A. y Ortiz de Zárate, A. (2014). Alternative Evaluation of a Traditional Oral Skill Assessment Tool in an English Teaching Program. LEC Journal, 1, 21-36. Lizasoain, A.; Ortiz de Zárate, A.; Véliz, M.; Luci, A. y Rojas-Mora, J. (2016). Evaluación de It's my Turn, una herramienta de autoaprendizaje de Inglés como Lengua Extranjera para contextos rurales de Chile. Revista Calidad en la Educación, 44, 98-128.

Mar-Molinero, C. (2000). The Politics of Language in the Spanish-Speaking World. Londres y Nueva York: Routledge.

- (2006). Forces of Globalization in the Spanish-Speaking World: Linguistic Imperialism and Grassroots Adaptation. En C. Mar-Molinero y M. STEWART (eds.). Globalization and Language in the Spanish-Speaking World. Macro and Micro Perspectives (pp. 8-26). Nueva York: Palgrave.

Martin, A. (2016). Second Language Teacher Education in the Expanding Circle: The EFL Methodology Course in Chile. Colombian Journal of Applied Linguistics, $18(1), 24-42$. <https://doi.org/10.14483/calj.v18n1.9471>

Martin, A. y Rosas-Maldonado, M. (2019). «Nothing to Do with Reality»: English Teachers' Perceptions of their Methods Preparation. Mextesol Journal, 43(3). Recuperado de <http://www.mextesol.net/journal/index.php?page=journal\&id article $=11942>$.

Matear, A. (2007). Tensions Between State and Market in Chile: Educational Policy and Culture. European Review of Latin American and Caribbean Studies, 83, 61-82. <https://doi.org/10.18352/erlacs.9633>

- (2008). English Language Learning and Education Policy in Chile: Can English Really Open Doors for all? Asia Pacific Journal of Education, 28(2), 131-147. <https://doi.org/10.1080/02188790802036679>

McKay, S. (2003). Teaching English as an International Language: The Chilean Context. ELT Journal, 57(2), 139-148. <https://doi.org/10.1093/elt/57.2.139>

Ministerio DE EDUCACIÓN (2014). Estándares orientadores para carreras de pedagogía en inglés (julio). Recuperado el 30 de noviembre de 2019, de <https:/www.cpeip. $\mathrm{cl} /$ wp-content/uploads/2019/03/Est\%C3\%A1ndares_Ingl\%C3\%A9s.pdf>.

- (2015). Bases curriculares $7^{\circ}$ básico a $2^{\circ}$ medio. Recuperado de $<$ https://media. mineduc.cl/wp-content/uploads/sites/28/2017/07/Bases-Curriculares-7\%C2\% BA-b\%C3\%A1sico-a-2\%C2\%BA-medio.pdf>.

- (2018). Beca vocación de profesor (febrero). Beneficios estudiantiles de Educación Superior. Recuperado el 4 de febrero de 2018, de <https://portal.beneficiosestu diantiles.cl/beca-vocacion-de-profesor-introduccion $>$.

- (2020). Programa Inglés Abre Puertas. Recuperado el 16 de junio de 2020, de <http:// ingles.mineduc.cl/programa-ingles-abre-puertas/>.

Nunan, D. (1992). Research Methods in Language Learning. Cambridge: Cambridge University Press.

Ortega, M. (2014). Assessing Trainees' Oral Performance in a Chilean Teacher Training Program: A Corpus-based Approach. Colombian Applied Linguistics Journal, 16(1), 10-16. <https://doi.org/10.14483/udistrital.jour.calj.2014.1.a01>

Riвosa, J. (2020). El docente socioconstructivista: un héroe sin capa. Educar, 56(1), 77-90. <https://doi.org/10.5565/rev/educar.1072> 
Ryan, G. y Bernard, R. (2003). Techniques to Identify Themes. Field Methods, 15(1), 85-109. <https://doi.org/10.1177/1525822X02239569>

Salado-Rodríguez, L.-I. y Ramírez-Martinell, A. (2018). Capital cultural en el contexto tecnológico: Consideraciones para su medición en la educación superior. RIES, 9(24), 125-137. <https://doi.org/10.22201/iisue.20072872e.2018.24.268>

Santelices, M.; Galleguillos, P.; González, J. y Taut, S. (2015). Un estudio sobre la calidad docente en Chile: El rol del contexto en donde enseña el profesor y medidas de valor agregado. PSYKHE, 24(1), 1-14. <https://doi.org/10.7764/psykhe.24.1.673>

The Commonwealth (2011). Women and the Teaching Profession: Exploring the Feminisation Debate. Londres: Commonwealth Secretariat / París: Unesco. Recuperado de <http://unesdoc.unesco.org/images/0021/002122/212200e.pdf>.

The World Bank (2019). Primary education, teachers (\% female). United Nations Educational, Scientific, and Cultural Organization (UNESCO). Institute for Statistics. Recuperado el 29 de noviembre de 2019, de <http://data.worldbank.org/ indicator/SE.PRM.TCHR.FE.ZS>.

Torche, P.; Martínez, J.; Madrid, J. y Araya, J. (2015). ¿Qué es "educación de calidad" para directores y docentes? Calidad en la Educación, 43, 103-135. <https://doi.org/10.4067/S0718-45652015000200004>

Valencia, E. y Manzi, J. (2011). Desempeño docente: Relaciones con antecedentes de los profesores y su contexto. En J. Manzi, R. González y Y. Sun (edits.). La evaluación docente en Chile (pp. 179-193). Santiago de Chile: Pontificia Universidad Católica de Chile.

VéLIz, M. (2007). The (Potential) impact of Mentoring on English Language Teaching in Chile at School Level. Literatura y Lingüistica, 18, 213-218. <https://doi.org/10.4067/S0716-58112007000100012>

Vera, O. (1942). Desarrollo histórico de los estudios oficiales de inglés en Chile. Anales de la Universidad de Chile. Recuperado de <http://www.anales.uchile.cl/index. php/ANUC/article/viewFile/24015/25338>.

Vivanco, H. (2016). Panorama histórico sobre la enseñanza de lenguas extranjeras en Chile. Lenguas Modernas, 47, 115-135.

Wagner, E. (2013). Survey Research. En B. Paltridge y A. Phakiti (eds.). Continuum Companion to Research Methods in Applied Linguistics (pp. 22-38). Londres y Nueva York: Bloomsbury. 